\title{
III. REPORTS
}

\section{Bericht über die Internationale Konferenz Hueber Polska Moderner Deutschunterricht - Erwerben der Fähigkeit zum Prüfungsbestehen oder des Wissens für das ganze Leben?, Warszawa 28. 08. 2010}

Die Internationale Didaktische Konferenz zum Thema Moderner Deutschunterricht Erwerben der Fähigkeit zum Prüfungsbestehen oder des Wissens für das ganze Leben? fand am 28. 08. $2010 \mathrm{im}$ Kyriade Prestige Hotel in Warschau statt. Organisiert wurde die Konferenz anlässlich des 10. Jubiläums des Verlags Hueber Polska, der Tochterfirma des deutschen Hueber Verlags.

Die Internationale Didaktische Konferenz wurde von der Geschäftsführerin des Verlags Hueber Polska, Joanna Ostendorf-Przedpelski eröffnet, die die Konferenzteilnehmer und die anwesenden Ehrengäste, darunter Michaela Hueber, Enkelin des Verlagsgründers und heutige Geschäftsführerin des Hueber Verlags, begrüßte.

Dann wurde Michaela Hueber gebeten, das Wort zu ergreifen. Frau Hueber ging in ihrer Rede auf ihren Großvater Max Hueber, den Verlagsgründer und ihren Vater, Ernst Hueber, ein. Sie skizzierte spannend die Geschichte des Verlags und erwähnte, dass gerade vor 10 Jahren das große Interesse an der deutschen Sprache in Polen zur Gründung von Hueber Polska führte.

Danach folgte der Plenarvortrag von Prof. Wilfried Krenn aus Wien, der zum Thema Multiple Intelligenzen sprach. Der Schwerpunkt des Vortrags war die Frage: Was hat Fremdsprachenlernen mit unterschiedlichen Intelligenzen zu tun? Der Redner beleuchtete zuerst, was unter dem Begriff Intelligenz in Anlehnung an Howard Gardner zu verstehen ist. Prof. Krenn listete des Weiteren multiple Intelligenzen auf. Abschließend plädierte Prof. Krenn dafür, dass multiple Intelligenzen Berücksichtigung im Klassenzimmer finden sollten.

Nach dem Plenarvortrag von Prof. Krenn begann die erste Runde der methodischen Workshops. Die Konferenzteilnehmer teilten sich in 3 Sektionen (A, B, C) auf. Jeder Teilnehmer konnte sich während der Konferenz insgesamt an zwei, vorher bei der Online- 
-Anmeldung gewählten, Workshops beteiligen. Insgesamt standen 6 anderthalb stündige Workshops in folgenden 3 Sektionen zur Wahl: Deutsch im Gymnasium (Sektion A), Deutsch für junge Erwachsene im Lyzeum bis zum Abitur (Sektion B) und Deutsch an Universitäten, Hochschulen und Sprachenschulen (Sektion C).

Da die Berichterstatterin an zwei Workshops (Workshop Nr. 2 und Nr. 6) teilgenommen hat, wird über diese zwei im Folgenden ausführlicher berichtet, die anderen werden dagegen nur kurz erwähnt.

In der Sektion A führte Wilfried Völker den Workshop Nr. 1 zum Thema deutsch.com: Das neue Lehrwerk mit Mehrsprachigkeitseinsatz für Jugendliche. Näher an den Erkenntnissen der Mehrsprachigkeitsforschung.

Zur selben Zeit moderierte Anne Robert aus Hannover den Workshop Nr. 2 in der Sektion B. Dabei handelte sich um Schritte international: Das moderne Lehrwerk für junge Erwachsene. Lernen der deutschen Sprache für den Alltag und Beruf - nicht nur für die Prüfungen sondern fürs Leben. Nach der Begrüßung und kurzen Vorstellung wurden den Teilnehmern die Musterexemplare des Kursbuches Schritte international ausgeteilt, so dass sich alle Einblick in das präsentierte Buch verschaffen konnten. Dann brachte Anne Robert das Lehrwerk Schritte international den Teilnehmern näher. Präsentiert wurden neben dem Kurs- und Arbeitsbuch auch CDs, unter anderem jedoch ein interaktives Lehrerhandbuch auf CD-ROM und Whiteboard-Materialien sowie DVDs. Zweifelsfrei ließ sich die Mehrheit der Teilnehmer von der breiten Vielfalt des Lehrwerks Schritte international überzeugen.

Zur selben Zeit dauerte in der Sektion C der Workshop Nr. 3, von Andreas Tomaszewski geführt, der Ziel- das neueste Lehrwerk für Fortgeschrittene zum Hauptthema machte.

Als die 3 Workshops zu Ende gewesen waren, wurden die Gäste zum kleinen Buffet im Hotelfoyer eingeladen. Im schönen Ambiente des Hotels wurde den Teilnehmern die Gelegenheit geboten, Gespräche zu führen, Anmerkungen nach Workshops zu besprechen oder sich über die Hueber-Neuerscheinungen zu informieren.

Nach der Kaffee-/Mittagspause begann die zweite Runde der Workshops.

Die an Deutsch im Gymnasium interessierten Lehrer beteiligten sich im Rahmen der Sektion A an dem Workshop Nr. 4 zum Thema Ideen- das moderne Lehrwerk für Jugendliche. Erfolgreich unterrichten mit den Erkenntnissen der Entwicklungspsychologie. Dieser wurde selbst von Wilfried Krenn- dem Co-Autor des Lehrwerks Ideen durchgeführt.

Im Mittelpunkt des Workshops Nr. 5 in der Sektion B stand hingegen das Lehrwerk Ausblick- das Lehrwerk für junge Erwachsene für die Niveaustufen B1 bis C1.

Die Deutsch an Universitäten, Hochschulen und Sprachenschulen Unterrichtenden nahmen an dem Workshop Nr. 6 in der Sektion C teil. Dieser wurde von Wilfried Völker geführt. Im Mittelpunkt stand das Lehrwerk Alltag, Beruf \& Co. Die Teilnehmer bekamen die Musterexemplare verteilt, um Einsicht in das Lehrbuch zu gewinnen. Herr Völker beleuchtete den Aufbau der Lektionen und ergänzte seine Rede durch Beispiele aus seiner eigenen Praxis als DaF-Lehrer in Frankreich, Deutschland und Mexiko.

An der Konferenz beteiligten sich Glottodidaktiker aus ganz Polen, diejenigen, die sich beruflich und/oder wissenschaftlich mit dem Deutschlehren- und lernen beschäftigen. In Warschau trafen sich sowohl schon erfahrene Deutschlehrer, die mit den Lehrwerken des Hueber Verlags lange arbeiten, als auch diejenigen, die erst vorhaben, mit HueberMaterialien den Deutschunterricht zu gestalten. Die Konferenzsprache war Deutsch. 
Zusammenfassend kann gesagt werden, dass die Konferenz Hueber Polska zweifelsohne vielen Konferenzteilnehmern neue Anstöße zur erfolgreichen, didaktischen Arbeit gab.

Abgeschlossen wurde die Konferenz mit der Verlosung von Geschenken. Die Veranstalter äußerten herzlichen Dank für die Teilnahme an der Konferenz. Alle Konferenzteilnehmer wurden auch zu methodischen Workshops, die jedes Jahr rund um das Schuljahr stattfinden sowie zur Teilnahme an der nächsten Didaktischen Konferenz herzlich eingeladen. 